\title{
Germanica
}

\section{Le péché salvateur ou : les paradoxes de l'imposture dans Le Cavalier suédois de Leo Perutz}

Die Sünde, die zu erlösen vermag oder: Die Paradoxien der Täuschungen

\section{Evelyne Jacquelin}

\section{OpenEdition}

\section{Journals}

Édition électronique

URL : http://journals.openedition.org/germanica/1791

DOI : 10.4000/germanica. 1791

ISSN : $2107-0784$

Éditeur

Université de Lille

Édition imprimée

Date de publication : 31 décembre 2004

Pagination : 105-118

ISBN : 9782913857148

ISSN : 0984-2632

Référence électronique

Evelyne Jacquelin, « Le péché salvateur ou : les paradoxes de l'imposture dans Le Cavalier suédois de Leo Perutz », Germanica [En ligne], 35 | 2004, mis en ligne le 05 octobre 2012, consulté le 06 octobre 2020. URL : http://journals.openedition.org/germanica/1791 ; DOI : https://doi.org/10.4000/ germanica.1791

Ce document a été généré automatiquement le 6 octobre 2020 .

(C) Tous droits réservés 


\title{
Le péché salvateur ou : les paradoxes de l'imposture dans Le Cavalier suédois de Leo Perutz
}

\author{
Die Sünde, die zu erlösen vermag oder: Die Paradoxien der Täuschungen
}

\author{
Evelyne Jacquelin
}

1 Si l'on cherche une figure majeure pour tenter de caractériser l'univers romanesque de Leo Perutz, écrivain autrichien de la première moitié $d u x^{e}$ siècle, on pourrait la trouver dans la récurrence de l'apparence trompeuse, des jeux de masques auxquels se prennent des personnages presque toujours condamnés à s'y perdre, mais aussi les lecteurs qui ne se méfieraient pas assez de récits souvent construits comme des leurres. Perutz échafaude des fictions où l'imposture se conjugue aux vertiges du dédoublement ou de l'amnésie pour faire éclater l'idée même d'une identité stable, comme l'ont noté la plupart des commentateurs, beaucoup rapportant cette thématique à la mise en question d'un "moi » que la "Jeune Vienne", dans le sillage d'Ernst Mach, avait comme l'on sait déclaré « insauvable » dès le début du $\mathrm{xx}^{\mathrm{e}}$ siècle$^{1}$.

Protagonistes et narrateurs, les mystificateurs ne manquent donc pas dans ces œuvres : le marquis de Bolibar, dans le roman éponyme, est un virtuose de la métamorphose dont les pouvoirs d'imitation atteignent à une perfection faisant passer le frisson de l'inquiétante étrangeté ; Turlupin met en scène la figure plus bouffonne d'un pauvre diable qui endosse un peu par hasard les habits de la noblesse ; dans un autre registre, la fin du Maître du Jugement dernier fait du baron Yosch un faussaire dont les mémoires sont marqués du sceau de la duplicité, tandis que Stanislaus Demba, personnage central du Tour du cadran, arpente Vienne en dissimulant ses mains menottées et invente des stratagèmes toujours nouveaux pour ne pas être découvert. 


\section{Les travestissements du Cavalier suédois}

3 Parmi tous ces faussaires démoniaques ou ridicules, naïfs ou manipulateurs, le héros $d u$ Cavalier suédois occupe une place particulière. Ce roman, le dernier publié par Leo Perutz avant l'exil et la guerre, en 1936, se trouve en effet tout entier rythmé par les métamorphoses du personnage, dont la carrière se fonde sur un échange d'identité en forme de trahison. Résumons l'intrigue pour plus de commodité. Au début de l'année 1701, lors de la seconde Guerre du Nord, deux hommes se rencontrent aux confins de la Silésie et se lient d'amitié. Tout les oppose, mais l'adversité les réunit, les vouant l'un et l'autre au gibet: le premier n'a point de nom et, vagabond, vit de larcins; le second, Christian von Tornefeld, a déserté pour rejoindre les rangs suédois. Réfugiés dans un moulin, il leur faut payer leur hôte, un inquiétant meunier à la solde de l'évêque du lieu, au nom duquel il recrute une main-d'œuvre destinée aux travaux de force dans les forges épiscopales. Le voleur, gibier de potence, venait justement y chercher un ultime refuge, mais il accepte de se rendre d'abord à un château voisin, où vivent des cousins de Tornefeld, pour en ramener l'aide matérielle qui permettra à celui-ci de se libérer envers le meunier et de poursuivre son entreprise. Découvrant que le domaine de Kleinroop n'a plus désormais d'autre maître que Maria Agneta, jeune fille angélique acculée à la ruine par le baron von Saltza, le larron se rêve soudain en sauveur et en maître des lieux. Il renonce alors à se faire connaître comme messager de Tornefeld, à qui la demoiselle s'est naguère promise, et décide d'envoyer à sa place dans les forges de l'évêque ce compagnon d'infortune en qui il voit désormais un rival. Mais pour pouvoir véritablement jouer le rôle de Christian dans le cœur de la belle, il doit d'abord faire fortune : s'étant approprié par ruse le talisman du jeune noble, une Bible trempée du sang de Gustave Adolphe de Suède, il prend la tête d'une troupe de bandits avec lesquels il pille les églises deux années durant avant de venir se présenter à Kleinroop sous l'identité de Tornefeld. Il va épouser sa belle ingénue qui lui donnera une fille nommée Maria Christine : sept années paisibles et prospères vont ainsi s'écouler avant que ce bonheur familial ne soit remis en question par l'irruption d'anciens compagnons de brigandage et, en particulier, par la menace que fait peser Lies la Rousse sur son examant, le faux cavalier, qu'elle veut dénoncer pour se venger d'avoir été abandonnée. Acculé, l'usurpateur devra se réfugier dans l'anonymat des forges épiscopales, tandis que le vrai Tornefeld, enfin libéré et croisé de nouveau au vieux moulin, retrouvera sa Bible et un équipement pour aller se couvrir de gloire dans l'armée suédoise : les deux personnages mourront au même moment, l'un en soldat, l'autre en forçat.

4 Jean-Pierre Chassagne a montré ce qu'une telle intrigue devait à la tradition du roman picaresque (op. cit., p. 14 et 18 sq.). Le voleur partage avec le picaro une condition de paria dont il veut se défaire. Privé dès l'abord d'un patronyme stable qui marquerait son assise identitaire, il se prête mieux que tout autre aux jeux de travestissement, glissant de rôle en rôle pour forcer son destin et conquérir l'amour en même temps qu'une position sociale. Ce parcours détermine la composition du roman en quatre parties dont chaque titre renvoie à une identité provisoire et à une étape dans la vie du personnage, tour à tour désigné comme "Le voleur ", "Le brigand de Dieu ", "Le cavalier suédois » et enfin "L'homme sans nom ». Un tel cheminement permet un enchaînement de péripéties où la corruption du monde répond à l'obscurité du protagoniste qui lui tend en retour un miroir impitoyable. Mais que fait Perutz de ce modèle? Notons d'abord, à cet égard, que le personnage central comme la conduite de 
l'action présentent, au sein de son œuvre, une singularité paradoxale. Avec Prunellede-Verre, le capitaine amnésique autour duquel se noue, dans deux chapitres cadres, l'énigme de La troisième balle, le cavalier est le seul héros anonyme de cet univers plein d'aventures. Et contrairement au premier, il occupe pleinement cette place, apparaissant comme le moins dépersonnalisé des protagonistes perutziens parce que l'auteur lui accorde d'avoir prise sur son destin, ne fût-ce que pour quelques années. La question de la faute et du châtiment, qui traverse tous les textes de Perutz, se trouve donc posée avec une acuité particulière. Loin de trouver sa place dans le monde après bien des vicissitudes ou de s'en détourner pour mener une vie pieuse, comme le veut en général la tradition picaresque, le faux cavalier paiera l'audace avec laquelle il a conquis sa brève liberté au prix fort. Faut-il donc parler, avec Jean-Pierre Chassagne, d'un anti-roman picaresque, où l'usurpation fait figure de faute irrémissible dans un monde sans pardon?

Le problème est complexe car l'intrigue se feuillette en une série de scénarios où la morale se renverse sans cesse, chacun des personnages principaux se trouvant tour à tour sauveur et débiteur de l'autre tandis que la trahison elle-même finit par porter de beaux fruits. Perutz aime emmener son lecteur sur de fausses pistes et brouiller ses repères, tant narratifs qu'éthiques ${ }^{2}$. De ce point de vue, la première imposture du Cavalier suédois consiste en un véritable coup de force par lequel une mémorialiste, d'abord avancée sur l'échiquier de la fiction pour en asseoir le crédit, se trouve soudainement escamotée et remplacée par un conteur anonyme et omniscient qui ne s'embarrasse en aucune façon de prouver qu'il parle en connaissance de cause. Or l'histoire du cavalier est à proprement parler indicible, puisqu'elle s'inscrit dans une lacune, brèche ouverte à la croisée de stratégies narratives contradictoires. Elle est donnée dans un récit enchâssé introduit par un «Prologue » où sont présentés les mémoires de Maria Christine, aristocrate des Lumières dont l'enfance fut assombrie par l'étrange disparition de son père. Comme le veut habituellement ce topos, la fiction éditoriale de souvenirs publiés par une grande dame ayant croisé, parmi d'autres célébrités du temps, Crébillon fils, Réaumur ou Jean-Sébastien Bach, semble d'abord avoir pour fonction de donner une plus grande vraisemblance et une dimension historique au roman. Mais le texte ne suivra pas cette voie puisque le prétendu récit de la mémorialiste n'est qu'un point de départ incomplet. Ce que le narrateur inconnu se propose de raconter à sa place, c'est précisément ce qu'elle-même ne sait pas, la réponse à ce mystère qui a pesé sur son enfance : comment son père pouvait-il se battre aux côtés de Charles XII de Suède jusqu'au fond de l'Ukraine tout en lui rendant visite la nuit, clandestinement, dans leur domaine silésien? Vanité des références puisées dans la resserre des faits avérés : l'objet du récit, l'histoire qui « doit » être dite ${ }^{3}$, n'a point de garant. Elle est la suite imaginaire d'un témoignage fictif, la résolution par affabulation d'une énigme inscrite en trompe-l'œil dans la réalité historique, pure invention dévoilée comme telle dans une pirouette marquant le triomphe d'une littérature qui s'avoue sans détour jeu de travestissement. Entrons donc dans ce palais des glaces aux perspectives changeantes, pour y suivre les scénarios entrecroisés dans lesquels crimes et châtiments se trouvent sans cesse redistribués. 


\section{L'usurpateur comblé ou : le règne des fausses vertus}

6 Comme il l'annonce dès la fin de son "Prologue ", Perutz nous offre en fait de Cavalier suédois «l'histoire de deux hommes $»^{4}$ et c'est le faux Tornefeld que l'on va suivre. Son ascension repose sur la ruse, le mensonge, le parjure. Après avoir décidé de changer son destin pour "s'asseoir à la table des maîtres $~^{5}$, il lui faut d'abord reprendre la promesse qu'il a faite au meunier de partir avec lui pour les forges de l'évêque et se débarrasser de son compagnon tout en s'assurant la possession du talisman que détient ce dernier. Le voleur surmonte toutes ces difficultés d'un seul coup en proposant au meunier un échange, lui conseillant d'emmener à sa place le jeune Tornefeld qu'il va convaincre par ailleurs de partir en lui faisant croire que les soldats sont à ses trousses pour le pendre et que les forges lui offrent un abri sûr. Ajoutant un serment hypocrite à ces traitrises, il jure devant Dieu de porter lui-même au roi de Suède la Bible convoitée tout en se promettant secrètement de la garder par-devers lui, dût-il la défendre contre le diable en personne (SR, 98). De tels mots sont rarement prononcés en vain, chez Perutz, et le petit voleur prend peu à peu des traits démoniaques. Avant de pouvoir usurper l'identité de Tornefeld, il va se mettre à la tête d'une troupe de bandits de grands chemins à la faveur d'une première imposture dont le symbolisme marque, non sans ironie, sa progression vers le Mal. Les brigands sont cernés, sans espoir de fuite, et Ibitz-le-Noir, leur chef, se trouve à l'article de la mort quand le voleur arrive pour leur proposer son aide : dans son délire, Ibitz croit voir arriver le diable en personne, venu l'entraîner vers l'abîme, et demandant grâce, il lui désigne ses gens à emmener à sa place (SR, 111). Il ne reste plus au voleur qu'à se réclamer de cette investiture, en jouant sur les mots, pour se faire obéir et conduire la troupe non en enfer, mais vers la liberté. Et comme il ne craint pas de porter la main sur les biens consacrés que recèlent les églises, échappant toujours comme par magie à ses poursuivants, on raconte bientôt que Satan a pris la tête de la bande (SR, 127 ; voir aussi SR, 157).

7 Le voleur de poules fait donc son chemin dans une totale immoralité, comme il le confie lui-même à son second, lui enseignant qu'« il est impossible d'arriver sans commettre un seul péché6.» Cependant, si la leçon paraît cynique, c'est au sens premier du terme qu'il faut songer, car en fait de morale, ce sont d'abord les conventions et les hiérarchies sociales que bouscule notre larron, débusquant l'hypocrisie de la noblesse et du clergé dont les idéaux se révèlent être de vaines paroles : sous les rodomontades transparaît la couardise, le goût du luxe et du lucre emporte tout scrupule et la sécheresse de cœur fait office de vertu apostolique. Les richesses temporelles de l'église sont ainsi dénoncées, ce qui permet de légitimer le pillage auquel se livrent les brigands (SR, 123-124), et le voleur, peut-être inspiré par Saint Laurent, fait la leçon à un curé qui l'a surpris, lui rappelant que les objets précieux offerts à sa paroisse par le seigneur du lieu ne sont rien en regard des dons faits aux pauvres, seuls à s'adresser véritablement à Dieu (SR, 130). Le portrait de l'évêque en maître de forges s'inscrit dans la même perspective. Le meunier défend la double identité de son employeur, prince lorsqu'il roule carrosse, mais homme simple et pieux quand il se rend à la messe, ce à quoi le voleur rétorque par une question impertinente, soulignant l'antinomie qui oppose à ses yeux les deux fonctions : «Et quand le diable emportera le prince séculier, qu'adviendra-t-il de l'évêque ? $»^{7}$. Une fois de plus, la formule n'est pas gratuite car le faste dont le prélat estime devoir s'entourer le conduit à exiger toujours plus d'une main-d'œuvre employée dans des conditions dignes d'un bagne, ce qui lui vaut 
justement le surnom d'« ambassadeur du diable » (SR, 200-201). À Malin, Malin et demi, pourrait-on dire : s'il est un lieu véritablement infernal, dans Le cavalier suédois, c'est bien ce domaine épiscopal précapitaliste, fabrique d'armes pour tous les partis en guerre sans préjudice de leur confession, placé sous le signe du feu éternel, avec ses fours à chaux et autres hauts fourneaux qui dévorent les vies de servants condamnés à disparaître dès lors que leur force de force de travail s'épuise. Aussi les forges, apparaissant comme le séjour de morts-vivants et d'âmes perdues (SR, 27, 200), sontelles plus souvent qualifiées d'« enfer de l'évêque » (SR, 27, 83, 201, 263).

Dans Le Cavalier suédois, le véritable Prince des ténèbres n'est donc pas celui que la superstition croit un peu trop vite débusquer derrière la malice d'un hors-la-loi. Il faut revenir, dès lors, sur cette figure anonyme et pourtant centrale du voleur, pour mieux comprendre de quelle étoffe il est fait. La scène d'exposition le présente à travers un portrait contrasté des deux " cavaliers». Ayant des origines radicalement différentes, ces compagnons d'infortune s'opposent aussi par leur expérience et leur tempérament. Tornefeld est un jeune homme plein de fougue, mais ses discours sur l'honneur nobiliaire et la défense de la foi luthérienne s'effondrent dès qu'il se trouve privé des attributs de sa caste. Le fuyard en guenilles mis à nu par l'adversité, à la merci du froid, de la faim et des soldats qui le recherchent, ne possède ni le courage ni la persévérance qu'il faudrait pour rejoindre les rangs suédois et se montrer digne de ses ancêtres et du jeune roi héroïque qu'est Charles XII. Inversement, le voleur, ancien valet de ferme, n'a d'autre avenir que la misère et le gibet, d'autre projet que d'y échapper en se vendant à l'évêque, mais il est aguerri par une vie d'indigence, possédant un sens pratique et terrien qui lui permet de surmonter bien des difficultés. La rencontre tourne à l'avantage du second, qui sauve la vie du premier en l'empêchant de se laisser mourir dans la neige. Elle révèle aussi la vanité d'idéaux impitoyablement rabattus sur une réalité qui les dément ${ }^{8}$ : à la bravoure du roi de Suède, le voleur oppose ainsi, avec des accents brechtiens, les impôts qui écrasent le peuple (SR, 20), et il finira par faire avouer à Tornefeld que son valeureux lignage et ses nobles proclamations sont de peu de poids face à la mort tant redoutée (SR, 95).

9 Les premières pages du récit enchâssé installent donc une perspective de classe à laquelle se mêle vite un sentiment de révolte face à la duplicité d'un monde où le code d'honneur nobiliaire apparaît finalement comme une forme d'idéologie (SR, 25-26, 31). Le passage au château de Kleinroop va prolonger cette première scène jusqu'à ce que l'expérience de l'injustice transforme progressivement le petit voleur sans espérance en un aventurier désirant parvenir à son tour et prêt, pour cela, à sacrifier son compagnon. L'équipée le confronte en effet à toute la corruption du monde, résumée dans ce domaine où un régisseur vénal s'entend avec le baron Saltza pour laisser les choses aller à la ruine et contraindre ainsi la jeune propriétaire, orpheline depuis peu, à s'endetter et à vendre ses biens un à un, à moins de se résoudre au mariage. Aux yeux du voleur, cette duplicité bouleverse l'échelle des valeurs, puisqu'un noble chrétien ne craint pas de se faire usurier (SR, 54-55). En même temps, cette fourberie le révèle à luimême par contraste et il apparaît à la fois comme un cultivateur pragmatique et, dans "une étrange métamorphose », comme "le seul honnête homme au domaine »: il décide alors de " parler sans détour » au maitre des lieux, prenant pour la première fois de sa vie « le droit chemin » pour se présenter « sans peur » à la porte de la demeure 9 .

10 À ce point, la fatalité sociale se conjugue avec un cheminement plus personnel qui va entraîner notre vertueux larron sur des voies moins droites qu'il ne se le promettait. Au 
dernier moment, la peur de l'homme si souvent traqué s'empare de lui et c'est dans la clandestinité qu'il va pénétrer au domaine. Pris pour le voleur qu'il ne veut plus être, il pourrait facilement se faire connaître et se tirer d'affaire, mais l'apparition de la jeune Maria Agneta le bouleverse, perturbant aussi la simplicité de ses aspirations morales. Il se rend compte que ses avertissements ne seront guère utiles à la jeune fille, incapable de se défendre seule (SR, 72) et compense son impuissance de paria par un rêve tout aussi « étrange » que sa métamorphose en honnête homme, s'imaginant être l'élu qui pourra la sauver. Vertueux, il prie Dieu, cependant, de l'aider à résister à cette tentation et apprend alors qu'elle est fiancée à Tornefeld, comprenant du même coup que s'il parle, elle sera perdue sans retour, prête à tous les sacrifices pour le jeune homme : mû par une force obscure, il préfère alors braver la mort en s'avouant voleur (SR, 76-79) et se verra épargné sur intercession de la belle, le fouet remplaçant la potence. Le sentiment d'injustice et de révolte, l'aspiration à un amour salvateur et le désir de revanche sociale se fondent alors indissolublement, sous les coups, donnant naissance à une pensée «monstrueuse » qui vient relayer les deux premières étapes de cette étonnante mutation : le voleur, qui a plus d'honneur que Saltza et plus de courage que Tornefeld, décide de se faire gentilhomme pour prendre cette place vacante auprès de Maria Agneta, à la tête du domaine (SR, 82). Voilà qui va l'entraîner sur la voie du péché menant à sept années de bonheur finalement résorbées dans l'anonymat du bagne épiscopal et d'une mort solitaire.

\section{Le pécheur châtié ou : le solde des créances}

11 Le théâtre du monde présenté dans Le cavalier suédois a de quoi troubler : variation sur la vanité d'un ici-bas entièrement corrompu, il semble abandonner son héros aux dérives terrestres sans amarrer la société des hommes à aucune norme éthique sur laquelle régler son action. Les princes et les églises faillissent en trahissant une justice dont ils devaient être les garants. Pour autant, Perutz ne compense pas cette mise en cause des hiérarchies traditionnelles en dessinant un horizon d'utopie révolutionnaire, et il ne laisse au peuple que la voie d'une violence vouée à se retourner contre lui, puisque le seul choix possible est d'être miséreux ou hors-la-loi, donc condamné à l'exclusion. Mais au sein même de ce "chaos éthique privé de Dieu ${ }^{10}$, une loi intangible demeure, qui éclaire les tribulations du faux cavalier : il s'agit du principe de miséricorde, comme l'a montré Jan Christoph Meister en réponse aux analyses proposées par Hans Harald Müller ${ }^{11}$. Toute une trame de fautes et de dettes à la fois morales et financières ${ }^{12}$ lie les personnages dans un destin commun dont le ressort complémentaire est justement cette compassion entendue non comme un apitoiement facile sur le sort d'autrui, mais comme la capacité à s'engager, à payer de sa personne, pour lui venir en aide.

12 C'est sous ce signe que s'établit, dès les premières pages de l'histoire enchâssée, le compagnonnage des deux "cavaliers", puisque le voleur prend en charge la vie de Tornefeld au péril de sa propre sûreté (SR, 22, 25-26). En retour, le Suédois n'a que de vaines promesses à offrir et se décharge de sa dette envers ce paria comme, par ricochet, envers le meunier, en tirant à la légère des traites sur un parent dont il ne sait pas encore qu'il est mort (SR, 22, 30, 40). En outre, c'est encore le voleur qui doit aller chercher le secours familial à la place du jeune homme, trop pusillanime, celui-ci ne se référant qu'en dernière instance à une fraternité de façade, quand celui-là l'avait 
précisément sauvé au nom de cette même fraternité ${ }^{13}$. Les raisons pour lesquelles le vagabond accepte cette aventure qu'il pense être la dernière éclairent à leur tour une autre dette courant au nom de Tornefeld. L'ancien valet de ferme est en effet aiguillonné par la curiosité de connaître le propriétaire des champs si mal tenus qu'ils viennent tous deux de traverser (SR, 23-24, 42). Ce questionnement se déploie en une sorte d'énigme obsédante jusqu'à ce que la vérité se dévoile avec l'apparition de Maria Agneta, orpheline si facile à duper qui préfère en outre se laisser dépouiller par Saltza plutôt que de renier son engagement envers Christian (SR 73, 76-77), tandis que lui ne pense qu'à la gloire des armes et use de leurs souvenirs communs comme d'une simple lettre de créance destinée à assurer au voleur le succès de sa mission en lui permettant de se faire connaître sans contestation possible (SR, 43).

13 Le point de départ de toute l'action est donc constitué par une double dette de Tornefeld, contractée d'abord envers la fiancée qu'il oublie, puis envers le miséreux auquel il doit la vie. Ce dispositif donne au péché du voleur une nécessité tragique puisque lui seul parait en effet en mesure de sauver la jeune fille, comme il l'imagine dans son rêve éveillé, de sorte que toute action de sa part le mettra nécessairement en faute, soit envers elle s'il n'agit pas alors qu'il en voit la possibilité, soit envers Tornefeld qu'il doit évincer pour venir en aide à Maria Agneta. Il n'en reste pas moins que la trahison d'un compagnon d'infortune demeure le crime par excellence, comme le proclame aussi le Juge suprême apparaissant au voleur lorsque celui-ci repasse par le moulin juste avant d'aller se présenter à Maria Agneta dans ses habits d'emprunt, vision pouvant être interprétée comme un signe de l'au-delà autant qu'un fantasme né des remords constants du voleur (SR, 85, 103, 179). Et la nature du péché est clairement énoncée, dans cette scène comme dans d'autres : tromper Tornefeld, c'est refouler tout sentiment de pitié (SR, 85), effacer l'autre dans l'oubli (SR, 105), laisser son cœur geler et se pétrifier (SR, 94), voir s'y éteindre l'étincelle céleste (SR, 167), donc se retrancher soi-même d'une humanité définie, en dernière instance, par cette capacité à la compassion qui est aussi un reflet de la miséricorde divine.

Ces deux thèmes complémentaires de la pitié et de la dureté traversent tout le récit, donnant lieu à des variations dans des épisodes secondaires. Ainsi, le voleur renoncera à tuer les anciens compagnons qui l'ont retrouvé, instruit par l'exemple de sa fillette dont le cœur ne se ferme pas encore sous l'effet de la peur et de la colère (SR, 207-209). À l'angélisme de la mère répond l'innocence de l'enfant, toutes deux montrant au faux cavalier la voie de la miséricorde. Le premier mouvement de Maria Agneta offre face au voleur, auquel elle offre d'abord le couvert avant d'intercéder pour qu'on ne le pende pas, fut ainsi dicté par la pitié (SR, 78-79) ; en réciproque, c'est lorsqu'il proclame dans son sommeil qu'il l'a aimée au premier regard, se donnant à elle tout en gardant le secret de sa véritable identité, que l'image du faux cavalier viendra se fondre définitivement avec celle de Christian dans le cœur de sa femme (SR, 186-187).

Le dénouement de l'action est à son tour entièrement motivé par l'interaction de ces deux forces opposées que sont la compassion et la fermeture à l'autre, attitudes ellesmêmes liées aux créances ouvertes entre les divers personnages, qui vont être tour à tour apurées. En se faisant gentilhomme, le voleur n'a pas seulement usurpé l'identité du jeune Tornefeld, il en a aussi réitéré les fautes en abandonnant à son sort ce compagnon d'errance, puis Lies, qui s'était entièrement vouée à lui (SR, 155, 235). Lorsque sa fortune s'inversera, il lui faudra aller jusqu'au bout de l'imposture en assumant non seulement le nom du vrai cavalier et la position qui y est attachée, mais 
aussi le destin auquel le jeune noble se sentait appelé. Pour préserver l'honneur familial (SR, 220), il décidera donc, contre ses convictions les plus profondes, d'aller chercher la mort au service du roi de Suède (SR, 227) et, contraint de délaisser Maria Agneta, il devra se laisser accuser de sacrifier leur amour à la gloire des armes, endossant ainsi la faute première de Christian (SR, 222). Si cet abandon est accompli dans un esprit de sacrifice qui relève encore d'un amour salvateur, le sort du voleur va se trouver définitivement scellé par le principe inverse du calcul et de la trahison. Averti que l'attachement de Lies s'est mué en haine (SR, 217, 244), il choisit pourtant d'en appeler encore à cette flamme, prêt aux dernières extrémités en cas d'échec (SR, 236-237). Il va alors trouver en cette femme outragée un adversaire à sa hauteur: au cours d'un affrontement où chacun voit sa propre perdition dans les yeux de l'autre, elle réussit à le marquer au fer rouge avant de succomber elle-même, le condamnant à chercher refuge dans les forges de l'évêque. Ayant préféré une nouvelle trahison à une mort plus noble au champ d'honneur, celui qui se trouve désormais dépouillé de ses habits d'emprunts pour devenir un "homme sans nom » se voit définitivement exclu de la compassion humaine, conformément au jugement prononcé contre lui lors de sa vision, qui le condamnait à porter seul le poids de ses péchés sans jamais pouvoir en appeler à la pitié de personne. Ce verdict d'abord incompris, le bagnard anonyme va en mesurer la portée à la toute fin de son parcours, alors que le visage entrevu de Maria Agneta éveillera en lui le besoin brûlant de convertir un amour perdu, parce que fondé sur une imposture, en un véritable pardon (SR, 270): victime d'une chute fatale avant de pouvoir se présenter à elle, il sera définitivement privé des paroles de consolation qu'il en attendait et, suprême ironie, c'est un ancien compagnon de brigandage, moine défroqué, qui vient le confesser dans son agonie, non pour le délivrer de ses fautes, mais pour lui faire avouer où son or est caché. Lorsque paraîtra l'ange du Jugement, le mourant pourra simplement déclarer qu'il a désormais saisi, en l'éprouvant dans la douleur, le sens de la sentence.

\section{Conclusion - De l'imposteur au traître ou : Judas et la «faute bienheureuse »}

16 La plupart des commentateurs soulignent le pessimisme qui s'exprime dans cette mécanique narrative où les fautes s'enchaînent inéluctablement les unes aux autres, soigneusement pesées pour se compenser comme autant de dettes qui passent du vrai au faux cavalier, ce dernier restant finalement seul face au ciel, à l'heure de sa mort, plus démuni encore qu'au début de l'aventure puisqu'entre-temps, il a goûté à la félicité d'un amour pur avant de devoir y renoncer. Ce dénouement qui mène le héros vers un dépouillement absolu, symbolisé par son statut d'«homme sans nom», lui donne la dimension d'un archétype, incitant à voir dans cette condamnation à vivre et mourir en forçat anonyme une sorte de résumé de la condition humaine : Jean-Pierre Chassagne parle à cet égard d'un effacement du sujet qui apparaît comme une fatalité, Jan Christoph Meister souligne ce que le récit reprend du contexte baroque dans lequel se déroule l'action en faisant d'une culpabilité conçue comme ontologique le fondement même de l'identité, et Michael Mandelartz, rappelant lui aussi le lien établi entre libre action et faute, renvoie au pessimisme de Schopenhauer et de Nietzsche ${ }^{14}$.

Hans-Harald Müller soutient, lui, que les deux personnages déterminent leur propre avenir de manière autonome, l'un par ses ambitions guerrières, l'autre en se rêvant 
gentilhomme, et considère que dans cette œuvre, les choix éthiques constituent la prérogative exclusive d'un sujet abandonné à lui-même au sein d'un monde sans repèr $\mathrm{e}^{15}$. On pourrait aussi considérer que Le Cahier suédois met à l'épreuve ce paradoxe théologique, aussi fondamental dans le judaïsme que dans le christianisme, constitué par le double postulat de la toute-puissance divine et du libre arbitre humain, chacun de ces termes entrant dans le jeu narratif d'une manière parfaitement équivoque. Ainsi, le statut des visions qui semblent déterminer l'avenir du voleur demeure aussi ambigu que la scène du Jugement divin : on peut y voir l'expression de fantasmes que le sujet va tenter de réaliser tout autant que les signes envoyés par une Providence qui tirerait dès lors tous les fils de l'action. De même, les diverses allusions à l'omnipotence divine peuvent être interprétées comme de simples topoï, reprises ironiques d'une rhétorique baroque que le récit ne vient ni confirmer ni infirmer, laissant le lecteur face à une question ouverte.

Cette ambivalence prend un sens nouveau si l'on songe que le faux cavalier est un imposteur d'une sorte particulière, puisqu'il fait son chemin par la trahison, acte dont l'un des principaux modèles est le baiser de Judas. Or la figure de l'Iscariote porte cette contradiction entre l'omniscience divine et le libre arbitre humain à un point d'incandescence mythique: son péché est nécessaire et prédit; il fallait un scélérat pour que la Passion et la Rédemption puissent aller à leur terme, mais comme l'on sait, "malheur à celui par qui le scandale arrive $»^{16}$. On retrouve quelque chose de ce tragique dans le cheminement du faux cavalier, dont la trahison n'est pas seulement liée à un dilemme fatal, mais apparaît aussi comme nécessaire pour que s'accomplissent les destins de Tornefeld et de Maria Agneta, dont chacun reprend certains aspects de l'histoire christique. Christian, le bien nommé, est vendu par le voleur qui se libère ainsi de son propre engagement. Il va subir, dans les forges de l'évêque, un martyre qui le mènera à l'accomplissement de soi dès lors placé dans la position déicide. Par ailleurs, le faux cavalier sauve Maria Agneta, personnage dont le nom, lui aussi, fait signe, et qui se trouve de surcroît explicitement comparé à l'agneau sacrifiée ${ }^{17}$. Si ce héros obscur est le seul de ses personnages auquel Perutz donne un certain pouvoir d'action, c'est donc pour en faire un pécheur dont la faute est finalement salvatrice, à l'image de la felix culpa prêtée à l'Iscariote par certains exégètes ${ }^{18}$. C'est aussi le sens que revêt la scène des retrouvailles entre les deux compagnons, où Tornefeld, fier de s'être endurci dans l'épreuve du feu, décharge le voleur de sa faute en reprenant son identité et les attribut qui y sont liés.

Mais en inscrivant en filigrane la figure de Judas dans la trame du Cavalier suédois, Perutz joue aussi, pour les retourner, avec les clichés antisémites attachés à une certaine postérité du mythe, le traître par excellence devenant à la faveur d'un rapprochement phonique l'exemple même de la perfidie et de la cupidité attribuées à l'ensemble du peuple juif ${ }^{19}$. Or, la félonie la plus noire, une fois encore, n'est pas là où l'on pourrait l'attendre et c'est la noblesse chrétienne personnifiée par Saltza qui apparaît d'abord comme l'incarnation d'un Judas explicitement désigné, à travers le qualificatif d'usurier, comme le Juif abhorré (SR, 54-55, voir aussi SR, 75): tel est le traitre qui sacrifie l'agneau, tandis que le larron, instrument de la Providence, le sauvera. Perutz opère donc un clivage, attribuant au monde chrétien cette noirceur de l'Iscariote si souvent utilisée contre la communauté juive tandis que par la bouche du paria, il réinsère la question de la «faute bienheureuse » et de la miséricorde dans la tradition juive en lui faisant proclamer, avec un certain sens de la provocation, sa foi de « grain de poussière » indigne de contester les arrêts du Très-Haut qui a décidé de faire 
de lui un pilleur d'église ${ }^{20}$, avant d'invoquer David, lui aussi grand pécheur et pourtant racheté. L'enjeu de l'allusion est clairement souligné par la réaction de son interlocuteur, qui s'emporte et conclut : « Ne m'embrouille pas les idées avec ton David. Mais une chose est vraie, et j'y ai souvent réfléchi. Pourquoi Dieu n'a-t-il pas fait tous les hommes chrétiens? Pourquoi y a-t-il tant de Turcs et de Juifs? Il y a là quelque chose qui ne va pas $^{21}$.»

Aux yeux de Perutz, Le cavalier suédois, rédigé à l'époque de la montée du nazisme et du $\mathrm{III}^{\mathrm{e}}$ Reich, était un livre écrit pour l'Allemagne, celui auquel il tenait alors le plus ${ }^{22}$. On voit s'y dessiner une opposition entre un monde chrétien belliqueux et sans merci, fonctionnant sur le mode de l'exclusion, et une culture juive qui ne fait encore qu'affleurer dans le récit, mais apparaît comme le lieu de la véritable miséricorde. Le traître du Cavalier suédois, exclu de la confession, ne peut se tourner vers aucune instance religieuse capable de lui apporter une consolation dans son agonie, la place de l'intercesseur étant occupée, au choix, par un évêque démoniaque ou par un bandit de grands chemins. Mais «l'homme sans nom» entre finalement dans la compassion divine par une autre voie : contrairement au Judas du Nouveau Testament, abandonné par la grâce, assez désespéré pour renoncer au ciel et mourir en impie, il périt en cherchant la pitié et, comprenant le sens d'un Jugement qui devient dès lors un ultime enseignement, il retrouve le chérubin auquel il avait été confié et connaît par son intermédiaire une forme de rachat. Or la créature céleste, qui se tenait d'abord devant Saint Jean $(S R, 163,165)$, se mue en un ange de la mort puisé aux traditions juives ${ }^{23}$, qui va intercéder afin que soit exaucé le dernier vœu du mourant: Maria Christine apprendra donc qu'elle ne doit plus attendre les visites nocturnes de son père et, dans son incrédulité d'enfant, prie sans le savoir pour l'âme du disparu. Le récit avait débuté par sa biographie de grande dame, dans laquelle s'inscrit aussi, à travers cette postérité, le rachat social du faux cavalier ; il se referme sur l'acte de compassion qui marque son salut, prière adressée au ciel pour un paria anonyme.

\section{NOTES}

1. Voir notamment Dietrich Neuhaus: Erinnerung und Schrecken, Die Einheit von Geschichte, Phantastik und Mathematik im Werk Leo Perutz', Francfort/M., Bern, New York, Paris : Peter Lang, 1984, pp. 49-58; Reinhard Lüth : Drommetenrot und Azurblau. Studien zur Affinität von Erzähltechnik und Phantastik in Romanen von Leo Perutz und Alexander Lernet-Holenia, Meitingen : Corian-Verl. Wimmer, 1988, pp. 104-117 et 355-392 et « Leo Perutz und das Fin-de-Siècle. Zu den literarischen Anfängen des Romanautors Leo Perutz und ihren Wurzeln in der Wiener Literatur um 1900 », in Modern Austrian Literature. Journal of the International Arthur Schnitzler Research Association, vol. 23, n¹, 1990, pp. 35-53 ; Hans Harald Müller : Leo Perutz, Munich : Beck, 1992, pp. 112-125 ; JeanPierre Chassagne: Le Scepticisme dans l'œuvre de Leo Perutz, thèse de doctorat, Université de Grenoble III, Juin 1999, pp. 11-76; Arndt Krieger : « Mundus symbolicus » und semiotische Rekurrenz. Zum ironischen Spiel der Wirklichkeitssignale in Romanen von Leo Perutz. Diss., Düsseldorf, 2000 ; Berlin : Tenea, 2000. 
2. Voir à ce sujet les analyses de Hans Harald Müller, notamment les postfaces aux œuvres de Leo Perutz, dont il a dirigé la réédition pour les éditions Zsolnay, à Vienne.

3. «Die Geschichte des "schwedischen Reiters" soll nun erzählt werden. » Der Schwedische Reiter, Vienne : Zsolnay, 1936/1958, p. 15 (toutes les citations extraites de ce roman seront données dans cette édition sous le sigle SR).

$\mathrm{Au}$ sujet du jeu sur des sources historiques fictives, voir aussi la postface de Hans Harald Müller in Der Schwedische Reiter, Vienne, Darmstadt : Zsolnay, 1990, pp. 243-259, notamment 253 sq.

4. «Es ist die Geschichte zweier Männer. » Tels sont les premiers mots du narrateur inconnu après qu'il a déclaré son intention de raconter l'histoire du cavalier (SR, p. 15).

5. «Bin lange genug am Tische der armen Rotte gesessen, keuchte er. Jetzt will ich sitzen am Herrentisch. ", ibid., p. 82.

6. « [...] so kannst du auch zu guten Tagen nicht kommen ohne jegliche Sünd'. » ibid., p. 124.

7. « Und wenn der Teufel den weltlichen Fürsten holt», höhnte der Dieb, « wo bleibt dann der Bischof? ", ibid., p. 87.

8. Voir à ce sujet Michael Mandelarzt : « Die Herrschaft der Ökonomie. Leo Perutz' Der schwedische Reiter ", in Studies in Humanities, n²7, Faculty of Arts, Shinshu University (Japon), mars 1993, pp. 213-220, ici pp. 214-216.

9. «Eine sonderbare Verwandlung war in ihm vorgegangen. [...] Es schien ihm, als wäre er, der Dieb, hier auf dem Hof der einzige ehrliche Mann, und als ehrlicher Mann wollte er mit dem Gutsherrn sprechen. [...] Diesmal aber ging er den geraden Weg, zum erstenmal in seinem Leben ging er aufrecht und furchtlos auf die Haustüre zu, er war ein ehrlicher Mann, er wollte eintreten, hatte mit dem Gutsherrn ein gerades Wort zu sprechen. » SR, pp. 56-57.

10. «In der gottlosen chaotischen Welt [...] gibt es keinen Glauben, keine verbindlichen ethischen Normen, es waltet in ihr kein gerechter geistlicher oder weltlicher Herr, sondern der Zufall oder die Göttin Fortuna [...]. » Hans Harald Müller, op. cit., p. 247 (voir aussi ibid., pp. 256-258).

11. Jan Christoph Meister : "Der schwedische Reiter - Von der Schuld der Identität", in Brigitte Forster et Hans Harald Müller Éd.: Unruhige Träume - Abgründige Konstruktionen, Wien: Sonderzahl, 2002, pp. 143-159, ici pp. 153 sq..

12. Voir à ce sujet Michael Mandelarzt, op. cit., pp. 216-219.

13. " "Es ist ein Mensch des anderen Menschen wert", sagte Tornefeld eilig, denn er fürchtete, der Dieb könnt' anderen Sinnes werden. » Op. cit., p. 42. La remarque est à opposer aux réflexions du voleur qui ne parvient pas à abandonner dans la neige son encombrant compagnon parce que son honneur de vagabond lui commande de venir en aide à un frère de misère malgré ses rodomontades sur l'honneur nobiliaire (SR, pp. 26-27).

14. Jean-Pierre Chassagne : op. cit., pp. 20-22 ; Jan Christoph Meister : op. cit., pp. 152-157 ; Michael Mandelartz : op. cit., pp. 217-218.

15. Hans-Harald Müller : op. cit., pp. 256-259.

16. Mt. 18, 6 ; Lc 17, 1. Sur cette question, on peut consulter en première approche les articles réunis par Catherine Soullard dans Judas, Éditions Autrement, Paris, 1999.

17. "Jetzt war ihm alles klar. Und nun verstand er auch, warum auf diesem Gut niemand ehrlich war [...] "Ein kleines, armes Lämmchen, von dem nimmt sich jedermann leicht Wolle”, sagte er zu sich mit einem grimmigen Lachen und ballte die Fäuste [...]. » SR, pp. 70-71.

18. Catherine Soullard: Judas à l'épreuve de l'exégèse, op. cit., p. 38.

19. Catherine Soullard : Le mystère Judas, ibid., p. 11.

20. «Wenn es dem höchsten Gott gefallen hat, mich zu dem zu machen, der ich bin - wie darf ich Staubkorn mich auflehnen gegen Seinen Willen?» SR, p. 141.

21. "Mach mir mit deinem David den Kopf nicht wirbelig. Aber das eine ist wahr, und ich habe oft darüber nachgedacht. Warum hat Gott die Menschen nicht alle zu Christen gemacht? Warum gibt es soviel Türken und Juden? Da ist etwas nicht so, wie es sein sollt'. » Ibid., p. 142. 
22. Lettre à Paul von Zsolnay, 20/1/1948 et lettre à Hugo Lifczis, 4/1/1946, citées in Hans Harald Müller et Brita Eckert : Leo Perutz : 1882-1957; eine Ausstellung der Deutschen Bibliothek Frankfurt am Main, Wien, Darmstadt : Zsolnay, 1989, pp. 353-354 et 329.

23. On le retrouve dès le premier chapitre de La Nuit sous le pont de pierre dans un contexte purement juif. Voir à ce sujet l'article correspondant dans le Dictionnaire encyclopédique du judaïsme (Geoffrey Wigoder et Sylvie Anne Goldberg Éd.), Parsi : Cerf/Robert Laffont, 1996, pp. 68-69.

\section{RÉSUMÉS}

L'apparence trompeuse est une figure récurrente dans l'œuvre de Leo Perutz, où l'imposture caractérise tout autant des constructions romanesques en forme de leurre que des personnages à l'identité mal assurée. Le Cavalier suédois en offre un exemple singulier, avec un narrateur anonyme qui se substitue brutalement à une mémorialiste pour présenter dans un récit enchâssé, selon un schéma picaresque, l'ascension d'un autre usurpateur lui aussi privé de nom, qui prend la place d'un jeune noble dont il épouse la fiancée, devenant ainsi hobereau. Ce héros, bien qu'anonyme, est le moins dépersonnalisé de l'univers perutzien car il décide en partie de son destin, ce qui pose avec acuité la question de la faute et du châtiment, présentée selon des perspectives changeantes. Ainsi, les méfaits de l'imposteur font écho à la corruption d'un monde où nobles et prélats trahissent leurs idéaux, dégradés en discours idéologiques masquant des rapports d'exploitation. Mais au sein de ce chaos subsiste un repère, le principe de miséricorde, auquel seront mesurées les actions de personnages liés les uns aux autres par une suite de dettes et de fautes, la trahison du vrai cavalier par le faux permettant de sauver la fiancée que le premier avait oubliée. Cet enchaînement fatal renvoie au paradoxe théologique que constitue le double postulat du libre arbitre et de l'omnipotence divine, éminemment illustré par l'histoire du traître par excellence, Judas, dont la figure transparaît sous celle de l'imposteur : Perutz retourne la postérité antisémite de ce mythe contre le christianisme et rachète le faux cavalier de sa felix culpa en le confiant à l'ange de la mort venu de la tradition juive.

Der falsche Schein ist ein oft wiederkehrendes Motiv im Werk Leo Perutz', wo die Täuschung sowohl trügerisch gebaute Romane als auch Figuren mit unsicherer Identität kennzeichnet. Davon gibt Der Schwedische Reiter ein besonderes Beispiel mit einem anonymen Erzähler, der eine Memoirenschreiberin unvermittelt ablöst, um nach dem Muster des Schelmenromans in einer Binnenhandlung den Aufstieg einer ebenfalls namenlosen Gestalt darzustellen, dessen Erfolg auch auf einer Verdrängung beruht : Der Protagonist eignet sich den Platz eines jungen Adligen an, heiratet dessen Verlobte und wird so zum Gutsbesitzer. Unter den Perutzschen Helden ist dieser trotz seiner Anonymität sich selbst am wenigsten entfremdet, denn er kann sein Schicksal zum Teil selbst bestimmen. Dies stellt die Frage nach Schuld und Strafe in besonderer Schärfe, wobei sie in wechselnden Perspektiven dargestellt wird. So entsprechen die Übeltaten des Betrügers der Verdorbenheit einer Welt, in der Adlige und kirchliche Amtsträger ihre Ideale verraten und sie zu ideologischen Phrasen verkommenen lassen, die Ausbeutungsverhältnisse verdecken. Aber inmitten dieses Chaos bleibt als Richtschnur das Prinzip der Barmherzigkeit erhalten: Daran werden die Handlungen von Gestalten gemessen, die durch eine Folge von Schulden verschiedener Art miteinander verbunden sind, wobei der Verrat des wahren Reiters durch den falschen die Rettung der Verlobten ermöglicht, die ersterer vergessen hatte. Diese 
verhängnisvolle Verkettung verweist auf jenes theologische Paradoxon, das in der gleichzeitigen Annahme von freiem Willen und göttlicher Allmacht besteht und durch die Geschichte des Verräters par excellence, Judas', beispielhaft veranschaulicht wird, einer Figur, deren Züge hinter denen des Betrügers zum Vorschein kommen: Perutz kehrt die antisemitische Nachwirkung dieses Mythos gegen das Christentum um und erlöst den falschen Reiter von seiner felix culpa, indem er ihn dem der jüdischen Tradition entstammenden Engel des Todes anvertraut.

\section{AUTEUR}

\section{EVELYNE JACQUELIN}

Université d'Artois, Arras 